\section{Drs. Friend and Bennett reply}

\section{To the Editor:}

We are somewhat baffled by Dr. Wolfe, el al's allegation that our editorial critique $^{1}$ of their recent paper ${ }^{2}$ is part of an ongoing opposition to the 2010 American College of Rheumatology Preliminary Diagnostic Criteria for Fibromyalgia. This seems odd, given that we independently validated those criteria $^{3}$. Indeed, in contradistinction to Dr. Wolfe's claim, we reviewed that paper and recommended "acceptance with revision." Notwithstanding these facts, Dr. Wolfe went on to inaccurately criticize our paper's methodology, and had to retract his mistake in an Erratum published in Arthritis and Rheumatology ${ }^{4}$. Our editorial, solicited by the editor, provides constructive comments, and should be viewed in that light. This is the accepted scientific method for the progressive advancement of knowledge ${ }^{5}$.

Our editorial stated that the Polysymptomatic Distress Scale (PSD) relies on the 19-point Widespread Pain Index (WPI) component as its primary assessment of pain. Wolfe, et al retorted that the WPI pain count "is not a primary assessment of pain" and that "we do not want people to use the WPI to measure pain severity." Was this a misreading of the editorial? We did not say that the WPI is a primary assessment of pain, but that the PSD relies primarily on the WPI to assess pain. The 19-point WPI count is the dominant component of the PSD, with a total of 19 of 31 cumulative pain points devoted to the total PSD score of 31. It is weighted 6 times greater than either assessment of fatigue or waking unrefreshed. It is the dominant factor in the proposed 5 PSD severity categories, especially at the higher severity levels (Figure 1). Elsewhere, as our editorial cited, Wolfe, et al also wrote:

"We suggest that the distribution of the PSD represents an aspect of the human condition, i.e., some patients report more pain and distress and some less, and PSD can be seen as a broad continuous distribution" 6

It is contradictory to claim that the PSD can be used to measure "pain and distress," while also claiming that the WPI is not a measure of pain when it is the dominant component in the PSD.

Wolfe, et al also criticized us for stating that the WPI, by definition, underestimates pain in disorders with fewer pain locations such as migraine and gout because the WPI measures only a pain site's presence, not its intensity. In Figure 2, we compare the WPI pain count (0-19) with the Symptom Impact Questionnaire (SIQR) visual analog scale pain intensity $(0-10)$ and the Medical Outcomes Study Short Form-36 (SF-36) pain subscale for the Wolfe, et al proposed 5 severity categories using data from our 2013 criteria $^{2}$. The WPI pain count correlates $r=0.43$ with SIQR pain intensity and $\mathrm{r}=0.48$ with SF-36 Pain, indicating that the more pain sites, the greater the pain intensity, reflected by the positively increasing lines. Importantly, the mean pain intensity at lower PSD severity categories is considerably greater than would be indicated by the corresponding pain site number, with the gap between the 2 converging with higher severity categories and WPI numbers. For example, at "mild severity" a mean of only 2 WPI sites is associated with a pain intensity of about 5.0. This confirms our point that the WPI underestimates the intensity of pain for disorders with fewer pain counts.

Regarding Dr. Wolfe's comments that we might contemplate the specificity of items used in the 2013 criteria, such as stiffness, balance, and environmental sensitivity, we refer him to Figure 1D in our 2013 criteria paper $^{3}$. This figure shows a random forest importance plot, with the 4 most important clinical features being environmental sensitivity, tenderness, cognition, and stiffness.

Lastly, Wolfe, et al write that they "await data on the acceptance" of the Fibromyalgia Impact Questionnaire (FIQR) and the SIQR (the disease neutral version of the FIQR), as if these are totally new scales, whereas they are updates of the most widely used and accepted scale for assessing fibromyalgia (FM) activity, namely the FIQR. In an article recently published in The Journal, we described the results of a study that compared the PSD and the SIQR as measures of FM severity ${ }^{7}$. It should not be presumed that the PSD severity scale and preliminary 2010 criteria are the gold standards with regard to the assessment of FM severity and criteria. The time-honored scientific method of iterative critique and revision will inevitably lead to worthwhile refinements of these scales and criteria.
Means for PSD, WPI and SSS by Severity Categories

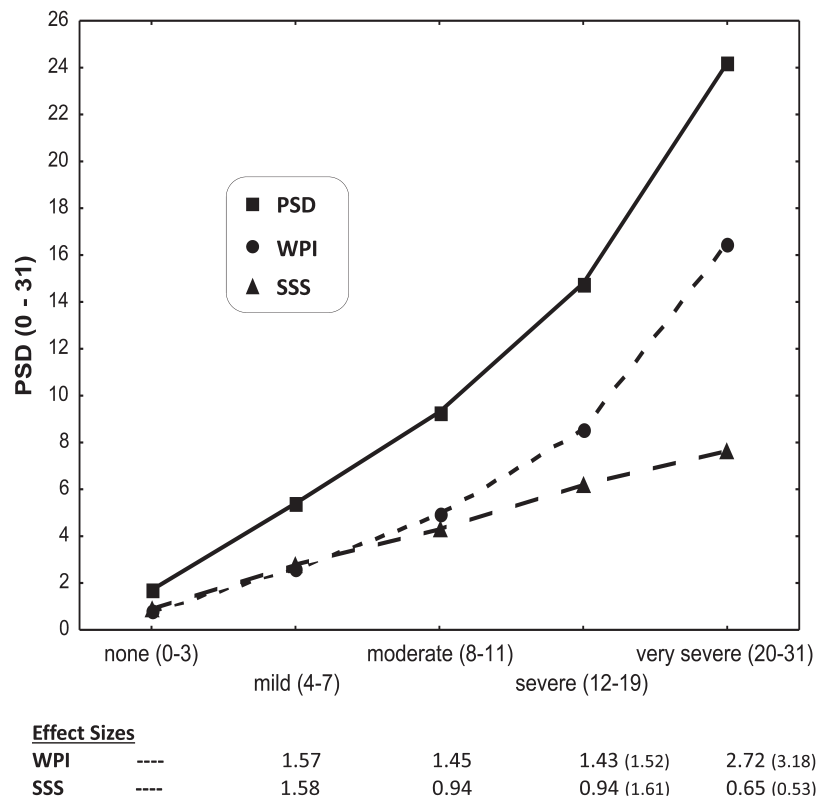

Figure 1. The comparison of the 3 lines (SSS, WPI, and PSD) starting at the moderate severity level shows that the WPI component is the dominant contributor to the PSD. The effect sizes (comparing a category mean with its preceding category mean) show that the SSS and WPI have equal contribution at the "none" and "mild" levels, but later the WPI becomes the dominant contributor to the PSD. Effect sizes for the SSS versus WPI also bear this out: $0.94,0.94,0.65$ (SSS) versus 1.45, 1.43, 2.72 (WPI). Because the WPI component exerts a disproportionately greater and uneven influence on the PSD, it has a different meaning at different severity levels, making the PSD neither easy to use nor simple to interpret, as stated in the Wolfe, et al letter. Values are taken from Tables 1 and 2 and the Supplemental Table in Wolfe, et $a l^{2}$. Effect sizes in parentheses are for the subset of patients in "severe" and "very severe" categories who are 2010 FM-positive (i.e., 3-6 WPI and $\geq 9$ SSS or $\geq 7$ WPI and $\geq 5$ SSS). SSS: Symptom Severity Scale; WPI: Widespread Pain Index; PSD: Polysymptomatic Distress Scale. From Friend and Bennett, J Rheumatol 2015;42:1364-7.

RONALD FRIEND, PhD, Professor of Psychology, Stony Brook University, Stony Brook, New York, and Adjunct Professor of Nursing, Oregon Health and Sciences University, Portland, Oregon, USA; ROBERT M. BENNETT, MD, Professor of Medicine and Nursing, Oregon Health and Science University, Portland, Oregon, USA. Address correspondence to Dr. R. Friend, 2347 NW Overton St., Portland, Oregon 97210, USA. E-mail: ronald.friend@stonybrook.edu

\section{REFERENCES}

1. Friend R, Bennett RM. A critical examination of the Polysymptomatic Distress Scale construct as a symptom severity questionnaire. J Rheumatol 2015;42:1364-7.

2. Wolfe F, Walitt BT, Rasker JJ, Katz RS, Häuser W. The use of polysymptomatic distress categories in the evaluation of fibromyalgia (FM) and FM severity. J Rheumatol 2015;42:1494-501.

3. Bennett RM, Friend R, Marcus D, Bernstein C, Han BK, Yachoui R, et al. Criteria for the diagnosis of fibromyalgia: validation of the modified 2010 preliminary American College of Rheumatology criteria and the development of alternative criteria. Arthritis Care Res 2014;66:1364-73.

4. Errors relating to study controls and criteria naming in the editorial by Wolfe (Arthritis Rheumatol, February 2015). Arthritis Rheumatol 2015;67:1645. 


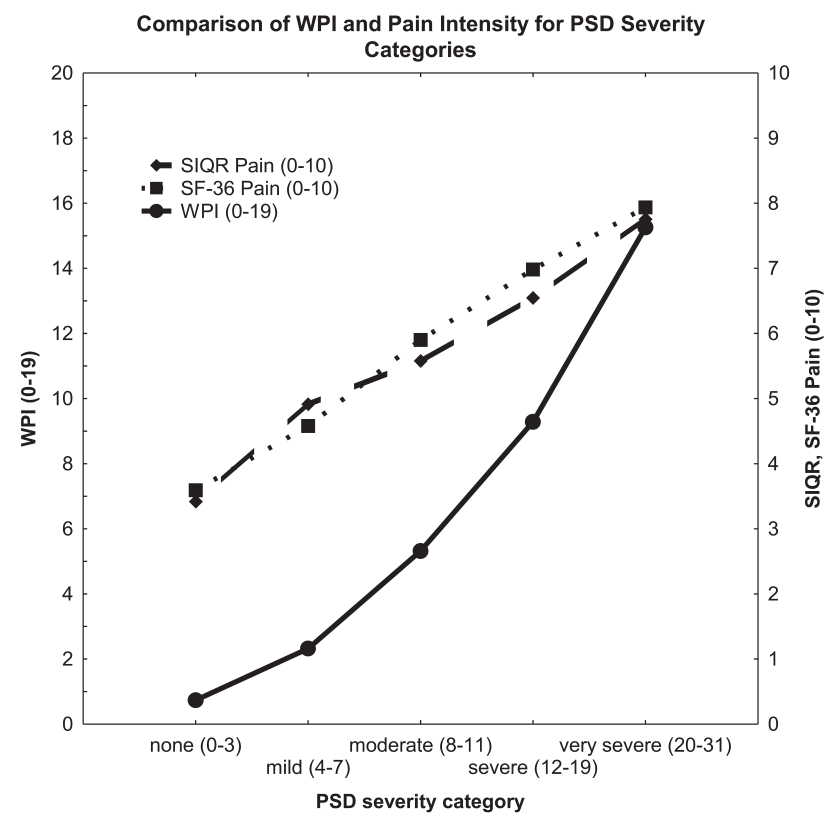

Figure 2. The SIQR Pain intensity (0-10; right Y-axis) and SF-36 Bodily Pain (scaled to $0-10$; right Y-axis) are higher than would be indicated by the corresponding low WPI scores (fewer pain locations; left Y-axis). Thus, the WPI underestimates pain intensity and SF-36 Bodily Pain at fewer pain locations. Pain intensity and pain locations converge with increased number of WPI pain locations. Data from Bennett, et al's sample of 321 patients with chronic pain that compared the 2010 and 2013 criteria $^{3}$. SIQR: Symptom Impact Questionnaire; SF-36: Medical Outcomes Study Short Form-36; WPI: Widespread Pain Index; PSD: Polysymptomatic Distress Scale.

5. Koop PM. Receiving feedback from reviewers: how to make the most of criticism. Can Oncol Nurs J 1999;9:148-50.

6. Wolfe F, Michaud K, Busch RE, Katz RS, Rasker JJ, Shahouri SH, et al. Polysymptomatic distress in patients with rheumatoid arthritis: understanding disproportionate response and its spectrum. Arthritis Care Res 2014;66:1465-71.

7. Friend R, Bennett RM. Evaluating disease severity in chronic pain patients with and without fibromyalgia: a comparison of the Symptom Impact Questionnaire (SIQR) and the Polysymptomatic Distress Scale (PSD). J Rheumatol 2015;42:2404-11.

J Rheumatol 2016;43:2; doi:10.3899/jrheum.151157 